\title{
Influence of Moisture Content on Defect Detection by Active Thermography Method to Concrete Structure
}

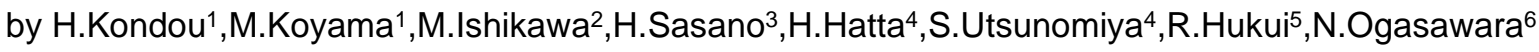 \\ ${ }^{1}$ Meisei University of engineering graduate course, 2-1-1 Hodokubo, Hino-shi, Tokyo, 191-8506, Japan, \\ ${ }^{2}$ Tokushima University, 2-24, Shinkuracho, Tokushima-shi, Tokushima, 770-8501, Japan, \\ ${ }^{3}$ Nihon University, 4-8-24, Kudanminami, Chiyoda-ku, Tokyo, 102-8275,Japan, \\ 4 Japan Aerospace Exploration Agency, 7-44-1, Jindaijihigashimachi, Chofu-shi, Tokyo,182-8522, Japan, \\ ${ }^{5}$ KJTD Co., Ltd. 9-29 Sumida 1-Chome Higashiosaka-shi,Osaka, 578-0912,Japan, \\ ${ }^{6}$ National Defense Academy, 1-10-20, Hashirimizu,Yokosuka-shi, Kanagawa,239-8686,Japan,
}

\begin{abstract}
At present, damage due to deterioration of concrete structures is a social problem. In order to prevent this problem, an infrared thermography method has been proposed and verified as a non-destructive inspection method different from conventional methods. Therefore, the infrared thermographic non-destructive testing considering with environmental condition and the weather condition which can happen to existing structure were considered in the present study. In this study, the effect of moisture content in concrete on defect detection was focused. As a result, it was suggested that the moisture content affects the defect detection.
\end{abstract}

\section{Introduction}

Inspection and evaluation techniques for concrete structures are essential to maintain the safety of civil buildings and infrastructure. In this study, we focused on the use of active thermography method to inspect concrete structures. This method is a non-contact method featuring shorter inspection time and covering a wider area than non-destructive inspection methods. The most conventional active thermography method is called pulse thermography (PT). In PT, the surface of a tested object is heated instantaneously by flash lamps. Then the surface temperature after that heating is monitored using an infrared camera. Subsurface defects appear as local hot or cold areas on the surface because the heat flow is disturbed by the defects. One of the techniques for improving the detectable defect depth is using pulse phase thermography (PPT). In PPT, temperatures obtained are transformed to phase by Fourier transform. Phase images are constructed from temperature images [1]. Past research has confirmed that it is possible to detect internal defects in concrete using PPT, Fig.1(a)(b). [2][3]. However, it is considered that the measurement environment greatly affects the practical use of infrared thermography. Therefore, in this study, a thermography method considering the environmental and weather conditions that can occur in existing structures was verified for application to actual structures.
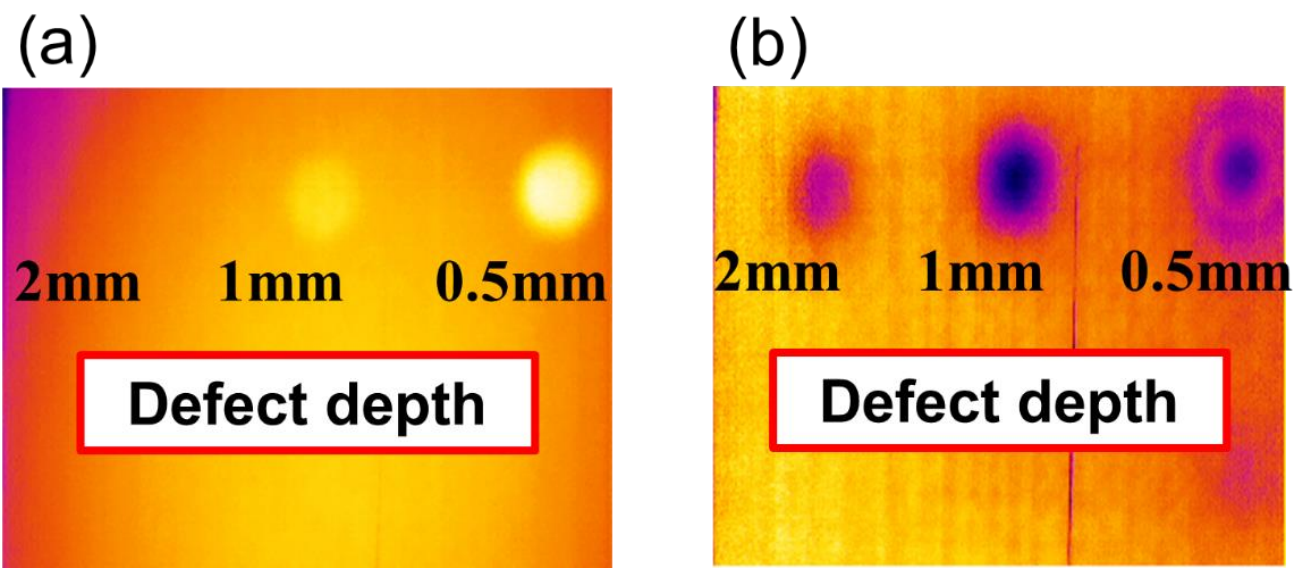

Fig. 1 (a) Thermal image, and Phase image. 


\section{Experiments}

Measurements were conducted heat input to the concrete surface using halogen lamp. In the test, the temperature difference of the surface temperature change in the defect part and the non-defective part was confirmed.

\subsection{Specimens}

The test piece was $300 \times 300$ in size and $100 \mathrm{~mm}$ thick, with artificial defects inserted at a depth of $50 \mathrm{~mm}$ from the surface. Specimen images are presented in Figs.2 (a) and (b).-The test piece was weighed after heating and drying, and then immersed in water for a predetermined time so that the test piece contained moisture. As a result of weight measurement after the impregnation with water, the water content was $1.47,10.99 \%$. Water content was calculated at gravimetric water content-
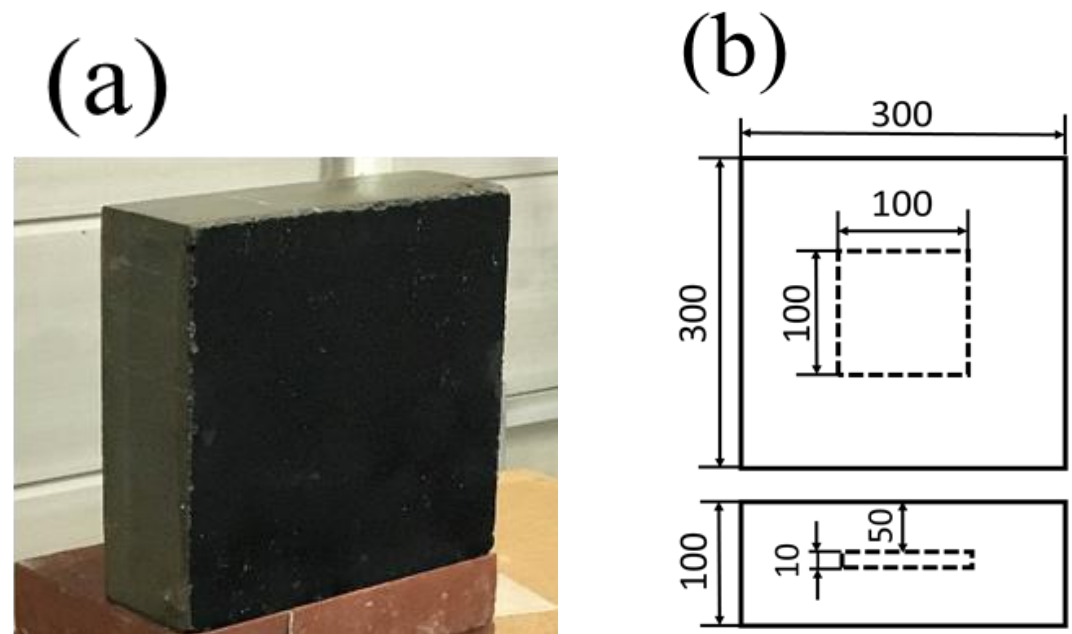

Fig.2 (a)Specimen image, and (b) Localizations of defects for specimen.

\subsection{Experimental setup}

Experimental setup is presented in Fig.3. In the experiment, the sample surface was heated with a halogen lamp for 200 seconds, and the temperature distribution on the surface during heating and cooling was observed using an infrared camera (A320; FLIR Systems Inc.) at a sampling frequency of $1 \mathrm{~Hz}$, Fig.4 (a) (b). The performance of the halogen lamp is presented in Table.1 and the power approximation performance curve is presented in Fig.5. Relationship between the maximum temperature difference and the total heat input on a $50 \mathrm{~mm}$ deep defect is presented in Fig.6. The inspection system used in the experiments was a Thermo Inspector (KJTD Co. Ltd.) [4].

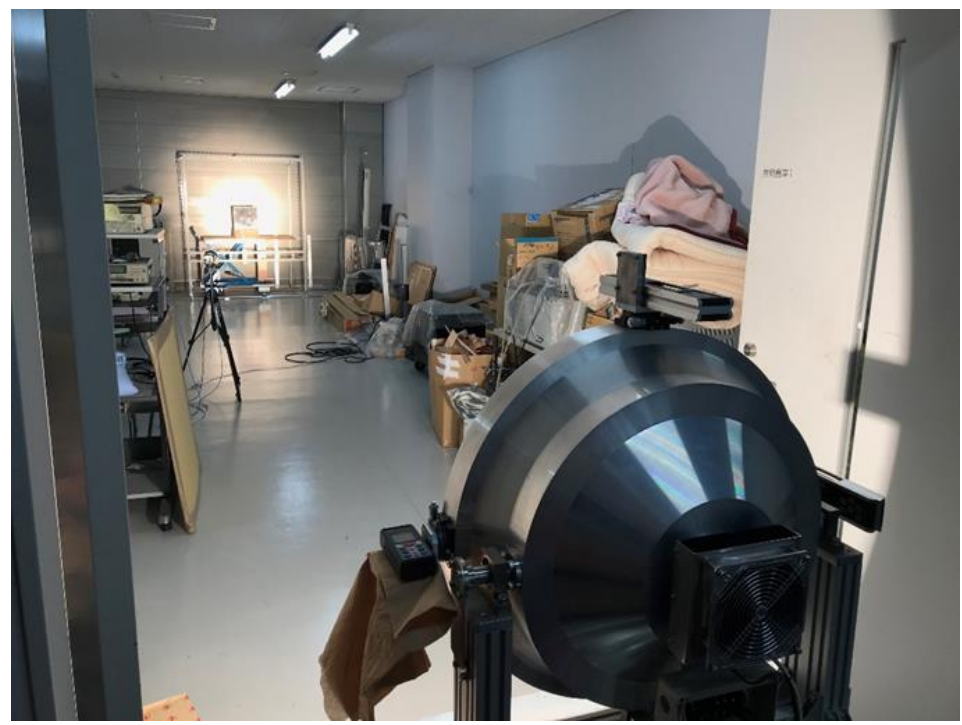

Fig.3 Experimental setup. 


\section{(a)}
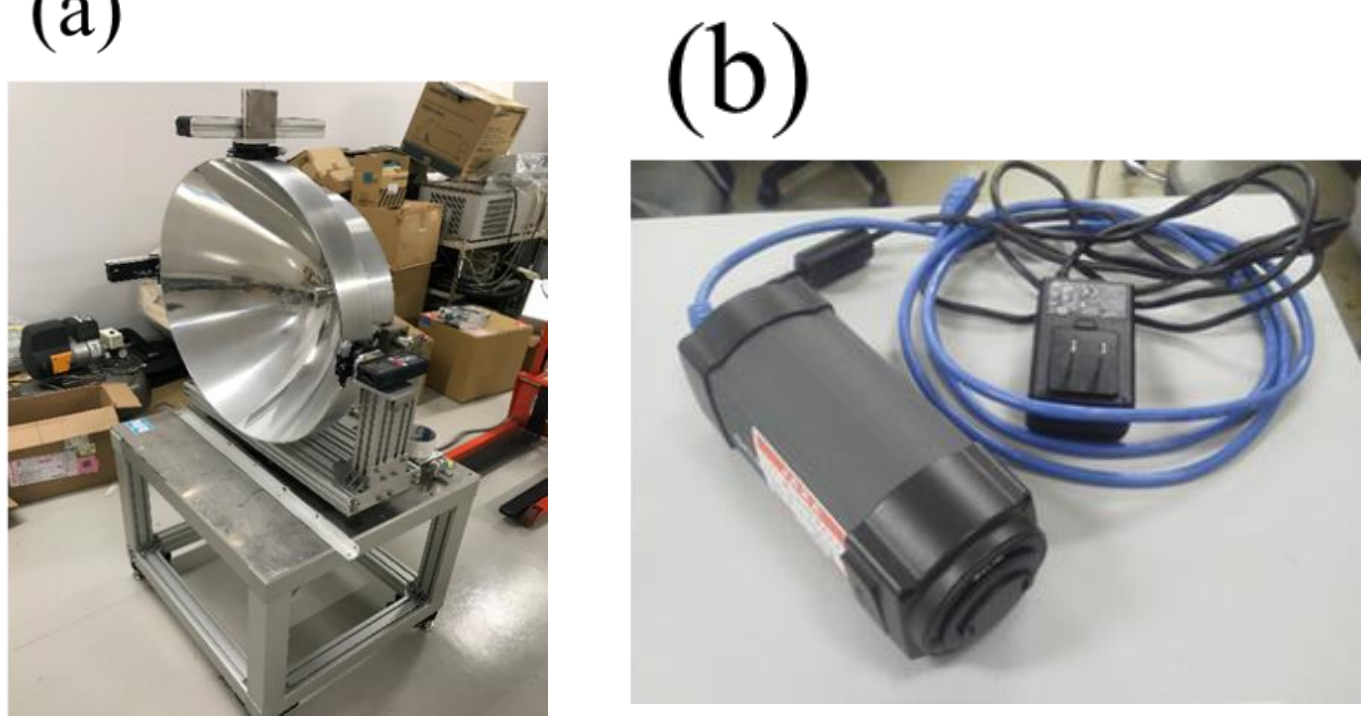

Fig.4 (a) Halogen lamp, and (b) Infrared camera (A320; FLIR Systems Inc).

Table. 1 Performance of halogen lamp.

\begin{tabular}{|c|c|c|c|c|}
\hline Irradiation distance $(\mathrm{m})$ & 5 & 10 & 15 & 20 \\
\hline Heat input $\left(\mathrm{W} / \mathrm{m}^{\wedge} 2\right)$ & 2432.97 & 891.82 & 454.11 & 256.23 \\
\hline
\end{tabular}

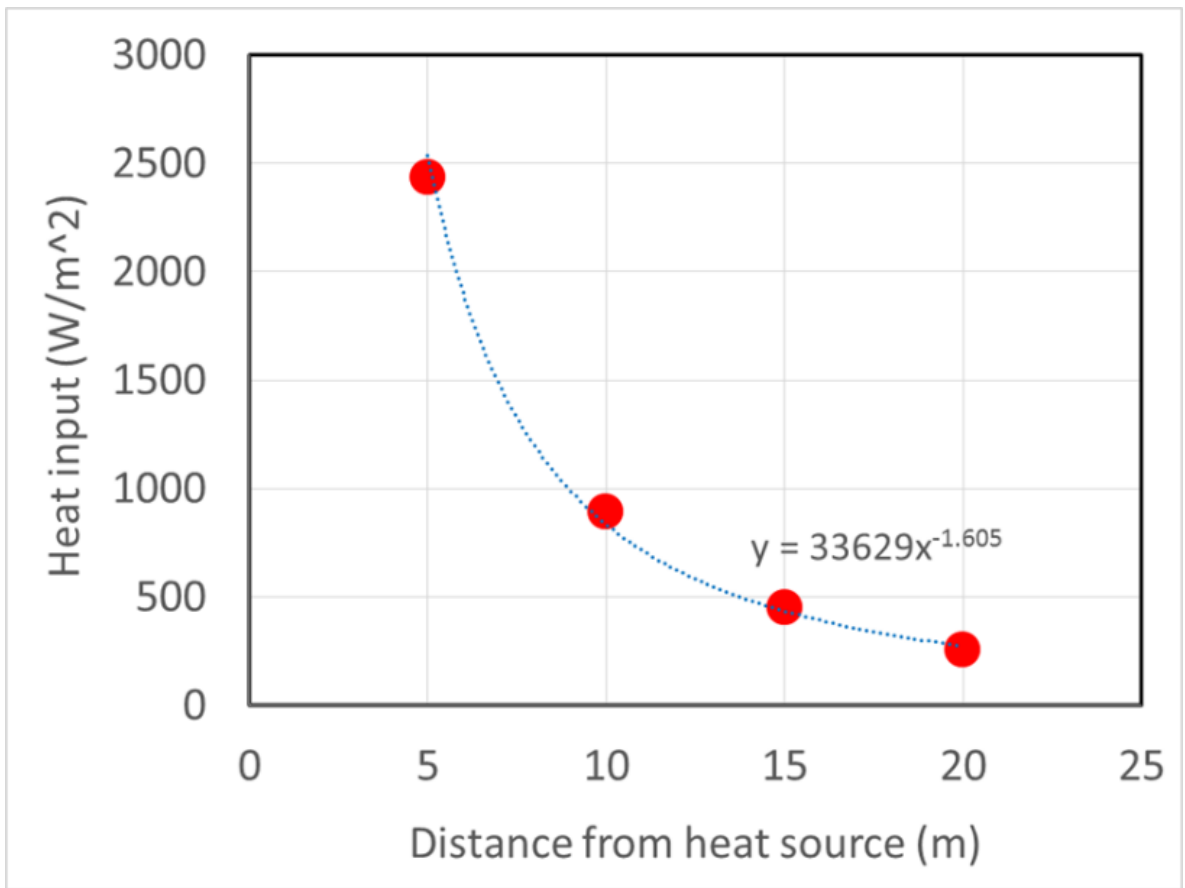

Fig.5 Power approximation performance curve. 


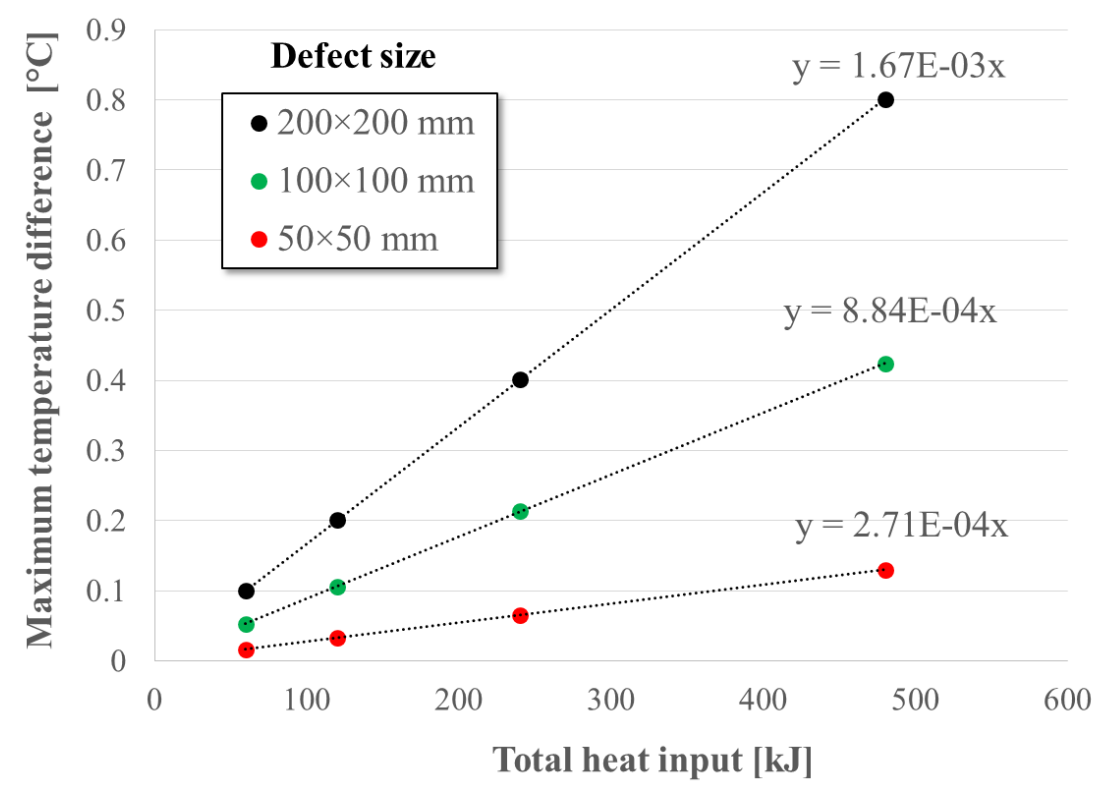

Fig.6 Relationship between the maximum temperature difference and the total heat input on a $50 \mathrm{~mm}$ deep defect.

\subsection{Experimental results}

Thermal images obtained are presented in Figs.7 (a), (b), and (c). The phase images obtained by Fourier transformation using thermal data are presented in Fig.8 (a), (b), and (c). Temperature difference between defective and non-defective areas on the defect with water contents are plotted in Fig.9. Temperature and phase images show that defects can be detected without being affected by moisture. And, it was confirmed that the temperature difference decreased with water content. It was confirmed that the temperature difference increased at the end of shooting compared to the dry state. Increase in temperature difference leads to improve in defect detection accuracy. The cause of this phenomenon is thought to be the change of the thermal conductivity and thermal diffusivity of concrete due to water content. As an index of thermal diffusivity, the heat given by the following Eq. (1) the thermal diffusivity $\alpha$ is used.

$$
\begin{gathered}
\alpha=\lambda /(\rho \times c) \cdots(1) \\
(\alpha: \text { Thermal diffusivity, } \lambda: \text { Thermal conductivity, } \rho: \text { density, } c: \text { Specific heat })
\end{gathered}
$$

In case of concrete physical property values ( $\left.\lambda=1.7(\mathrm{~W} / \mathrm{mK}), \rho=2240\left(\mathrm{~kg} / \mathrm{m}^{\wedge} 3\right), \mathrm{c}=800(\mathrm{~J} / \mathrm{kgK})\right), \alpha=0.95\left(\mathrm{~mm}^{\wedge} 2 / \mathrm{s}\right)$ is obtained. As well, in case of water physical property values $\left(\lambda=0.6(\mathrm{~W} / \mathrm{mK}), \rho=998\left(\mathrm{~kg} / \mathrm{m}^{\wedge} 3\right), c=4200(\mathrm{~J} / \mathrm{kgK})\right)$, $\alpha=0.14\left(\mathrm{~mm}^{\wedge} 2 / \mathrm{s}\right)$ is obtained. This calculation result shows that the heat transfer coefficient is reduced due to the inclusion of water. The reduction of the heat transfer coefficient hinders the heat transfer in the in-plane direction, and as a result, the temperature difference between the defective portion and the sound portion is reduced. It is considered that this phenomenon caused the decrease in defect detectability due to the water content. The details of the water content and thermal conductivity will need to be clarified by future experimental verification. From these results, it was suggested that the effect of moisture in concrete is an important issue when applying this inspection method to actual structures. 
(a)

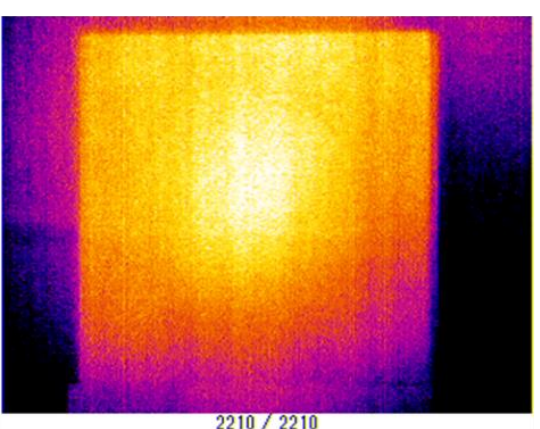

(b)

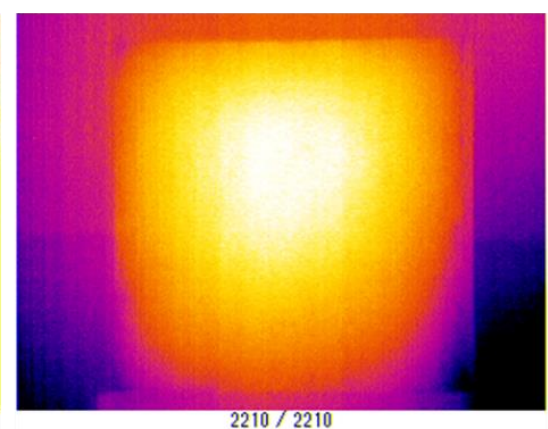

(c)

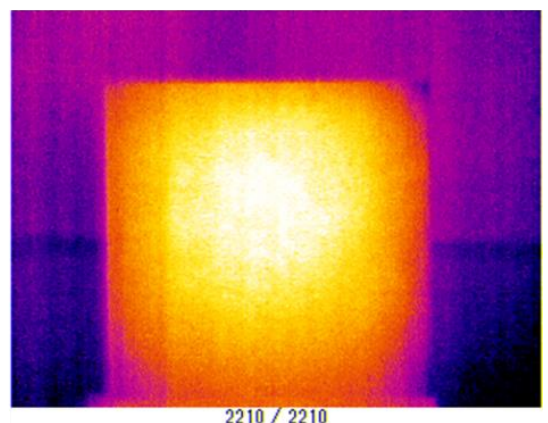

Fig.7 Thermal images obtained (a)No hydrous specimens, (b) water content 1.47\%, and (c) water content $10.99 \%$. Both are thermal images at 2210 s from start heating.

(a)

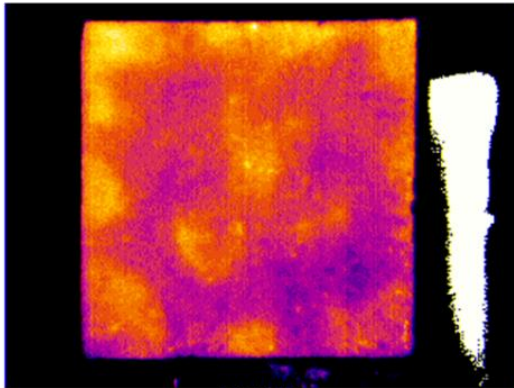

$0.0005 \mathrm{~Hz}$ (b)

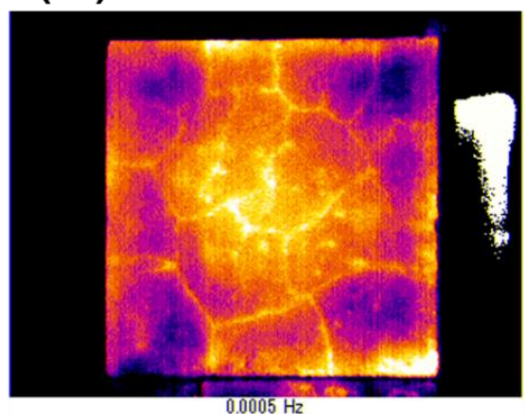

(c)

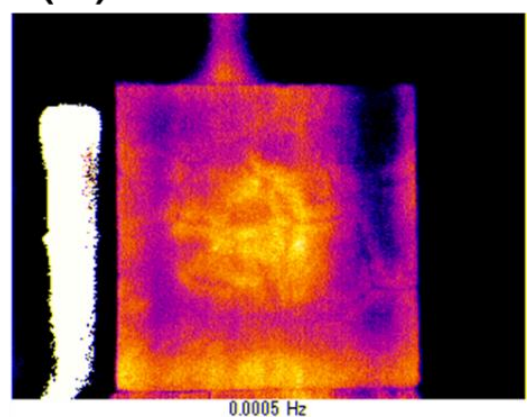

Fig.8 Phase images at frequency of $0.0005 \mathrm{~Hz}$ from thermal data obtained (a)No hydrous specimens, (b) water content $1.47 \%$, and (c) water content $10.99 \%$.

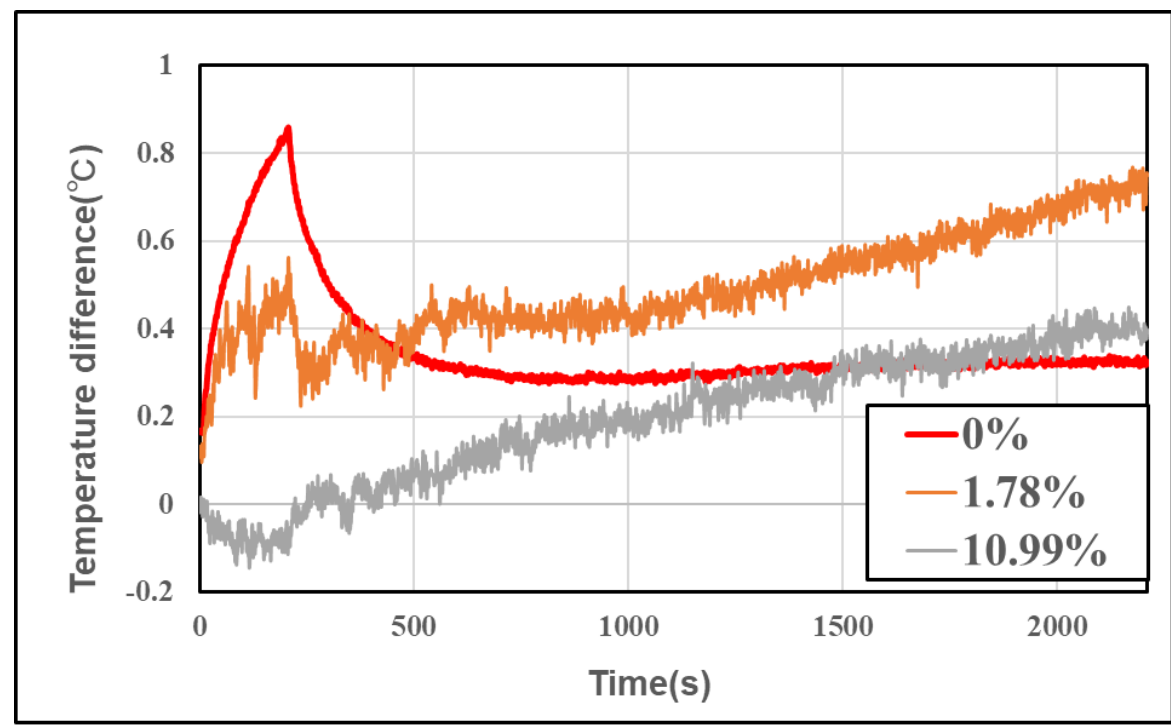

Fig.9 Experimental results observed temperature difference for no hydrous specimen, and water content 1.47, $10.99 \%$. 


\section{Conclusion}

Active thermographic inspection of hydrous concretes was undertaken using halogen lamp heating apparatuses. It was ascertained this experiment that there is difference the inspection capability of thermographic inspection when increased gravimetric water content. These results suggest that applying this method in a real environment requires data processing techniques that take into account the water content of the concrete.

\section{REFERENCES}

[1] Maldague, X. and Marinetti, S., Pulse phase infrared thermography, Journal of Applied Physics, Vol. 79, No. 5 (1996), pp. 2694-2698.

[2] F. Weritz, G. Wedler, A. Brink, M. Röllig, C. Maierhofer, H. Wiggenhauser, Investigation of concrete structures with Pulse Phase Thermography, Mater.Struct. 38 (2005) 843-849.

[3] Masashi Ishikawa,"Non-contact non-destructive inspection method by infrared pulse phase thermography method", ISAS RESERCHNOTE, 869, 2012

[4] KJTD Co.,Ltd. <https://www.kjtd.co.jp/products/thermo_inspector/index.html> 